\title{
Polarization as a Berry Phase
}

R. Resta

Dipartimento di Fisica Teorica, Università di Trieste

\section{The macroscopic polarization of a crystalline dielectric is best defined as a Berry phase of the electronic Bloch wavefunctions, a feature that is best exemplified by the spontaneous polariza- tion of a ferroelectric crystal for which a microscopic theory was previously unavailable.}

Although the macroscopic polarization is the basic quantity used to describe dielectric media, it has, surprisingly, long evaded precise microscopic definition. Elementary textbooks define the polarization as the dipole of a unit cell, or something similar. But such a picture is only acceptable if the polarization elements are assumed to be discrete. In a real dielectric, the electronic distribution is continuous (and often delocalized). So the dipole of a unit cell is poorly defined: in fact, macroscopic polarization has nothing to do with polarized dielectric.

Some important experimental facts are worth noting. The absolute polarization of a crystal in a given state has never been measured as a bulk property. Instead, well-known bulk properties are derivatives of the polarization with respect to suitable perturbations (permittivity, pyroelectricity, piezoelectricity, dynamic charges). In one important case (ferroelectricity), the relevant bulk property is inferred from the measurement of a finite difference in the polarization (see below). In all these cases, the derivative or the difference in the polarization is typically accessed via the measurement of a macroscopic current. For instance, to measure the piezoelectric effect, the sample is strained along the piezoelectric axis while being shorted out with a capacitor (see Fig. 1). This is an important clue to understanding the modern theory on the polarization of a crystal-

Fig. 1. An illustration of experimental arrangement used to measure the piezoelectric effect: the current flowing along a sample shorted out by a capacitor is measured while the sample is strained in one direction. the periodic charge distribution of the

line dielectric: in quantum mechanics the modulus of the wavefunction provides the charge density, whereas to obtain the current one needs the phase as well.

\section{The Modern Theory}

According to the modern theory [2], the macroscopic polarization is best defined (and calculated) as a Berry phase of the electronic Bloch wavefunctions. A definitive formulation of the theory was achieved in 1992-3 [3], and its most successful application is the spontaneous polarization of a ferroelectric crystal [4], for which a microscopic theory had previously been unavailable.

The theory of polarization based on a Berry phase applies to the case of zero temperature and zero electric field. It therefore only concerns phenomena where the macroscopic polarization is induced by a source other than an electric field. This is the case for ferroelectricity, piezoelectricity and lattice dynamics. The zerofield condition is essential in order to ensure that the Hamiltonian describing the crystal electrons is periodic - and its eigenfunctions have the Bloch form - even in the perturbed case. Whenever a macroscopic field is present, the potential is in fact no longer a periodic function of the crystal lattice.

To illustrate the concept of polarization in zero field, consider the specific example of the piezoelectric effect. In the typical experiment (Fig. 1) where the crystal is uniaxially strained whilst in a shorted capacitor, the piezoelectric tensor by definition measures the linear polarization induced by the macroscopic strain at zero field [5]: this polarization difference is observed via the current flowing along the wire. Under these circumstances, one is studying a bulk property of an open quantum system, where electrons are injected into one face of the crystal and drained from the opposite one.

This perspective helps one appreciate understanding the phenomenon. Quite generally, a non-trivial Berry phase may

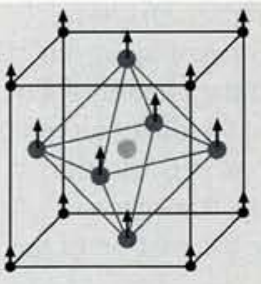

a

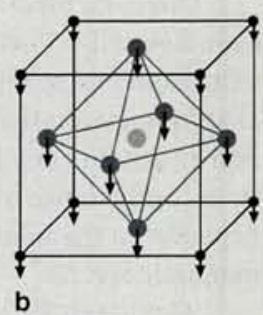

Fig. 2. Structure of the cubic perovskite $\mathrm{KNbO}_{3}$ : grey circles represent $\mathrm{Nb}$, black circles represent 0 , and small black circles represent $K$. The arrows indicate the ionic displacements (magnified by a factor of 4 ) in the ferroelectric tetragonal structure. Two of the possible enantiomorphous structures are shown.

only occur in a non-isolated quantum system, and originates from the interaction with the "rest of the universe" (see box). In the present example, the electrons in the crystal are interacting with an external apparatus and are "enslaved" by it. This interaction gives rise to a Berry phase in the electronic wavefunction, whose observable effects constitute the macroscopic polarization difference $\Delta P$ between the strained and unstrained structures of the crystal:

Other important examples which the modern theory of polarization deals with are dynamic charges (for lattice dynamics) and ferroelectricity. We focus on the latter phenomenon in the following. One of the simplest ferroelectric crystals is the perovskite $\mathrm{KNbO}_{3}$ (Fig. 2), with a cubic prototype phase above the Curie temperature. When the temperature is lowered, the material undergoes a transition to a tetragonal phase which is characterised by microscopic displacements of the ions from their high-symmetry sites. The distortion determines a preferred polarity of the tetragonal axis and is responsible for the occurrence of spontaneous polarization. A typical measurement of the spontaneous polarization is performed through polarization reversal (Figs. 2 and 3). The sample is placed in a capacitor and the transition between the two enantio-

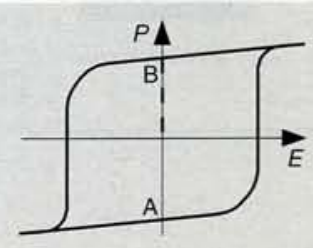

Fig. 3. A hysteresis loop for the measurement of polarization reversal in a ferroelectric. The magnitude of the spontaneous polarization $P_{s}$, as phenomenologically defined, is indicated by the dashed vertical segment. 
morphous structures A and B is driven by an applied electric field. The experimental setup measures the integrated macroscopic current flowing through the sample during the hysteresis cycle (Fig. 3). One half of the difference $P_{\mathrm{B}}-P_{\mathrm{A}}$ phenomenologically defines the magnitude $P_{s}$ of the spontaneous polarization. The best definition of the spontaneous polarization closely parallels the way this observable is measured: the spontaneous polarization of a given ferroelectric structure is the integrated current which flows through the crystal while the ions are continuously displaced from the centrosymmetric to the ferroelectric structure.

\section{How the Theory Works}

One can get an idea of how the theory works using the specific example of ferroelectricity, taking a one-dimensional model (Fig. 4). I show how the polarization problem fits into the general setting introduced by Berry: the polarization difference $\Delta P$ between two given structures of the crystal is a Berry phase, i.e. the loop integral in a suitable parameter space of a given linear differential form, called the Berry connection (see box). Without providing any proof, I only define the parameter $\zeta$ and I establish the closed path $C$ around which the loop integral is calculated.

One first defines a scalar parameter $\lambda$ which measures the amplitude of the ferroelectric distortion in dimensionless units for the centrosymmetric structure (Fig. 4, upper panel: $\lambda=0$ ), and for the ferroelectric structure (Fig. 4, lower panel: $\lambda=1)$. For any value of $\lambda$ the electrons move in a lattice-periodic potential $V^{(\lambda)}(x)$, with period $a$. In the simplest model insulator there are only two electrons per cell, and hence one doubly occupied band whose wavefunctions have the form:

$$
\psi^{(\lambda)}(k, x)=\mathrm{e}^{i k x} u_{k}^{(\lambda)}(x)
$$

where $k$ is the Bloch vector in the onedimensional Brillouin zone $[-\pi / \mathrm{a}, \pi / \mathrm{a}]$, and $u_{k}^{(\lambda)}(x)$ is a periodic function of $x$ with

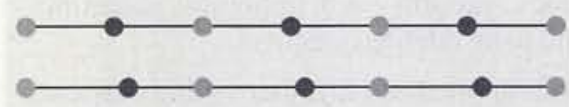

Fig. 4. An illustration of a one-dimensional ionic crystal with a centrosymmetric structure (upper) and a brokensymmetry ferroelectric structure (lower). Grey and black circles represent cations and anions, respectively. Comparing with Fig. 2, this linear chain mimics a single vertical line of niobium and oxygen atoms.

\section{The Berry Phase}

The Berry phase is a very general concept, having observable manifestations in several different areas of physics [1]. One starts with the most generic quantum Hamiltonian having a parametric dependence:

$$
H(\xi)|\psi(\xi)\rangle=E(\xi)|\psi(\xi)\rangle
$$

where $\xi$ is defined in a suitable domain: a two-dimensional $\xi$ with real values has been chosen for illustration in the figure. In the original Berry paper $H(\xi)$ is the electronic Hamiltonian of a molecule in the Born-Oppenheimer approximation, and $\xi$ is a nuclear coordinate. But the theory is quite general and applies to the most disparate parametric dependence: in the theory for the macroscopic polarization of a crystalline dielectric the parameter $\xi$ is a rather exotic one (Fig. 5).

We assume that $|\psi(\xi)\rangle$ is the ground state, non-degenerate for any $\xi$. The phase difference $\Delta \varphi_{12}$ between the ground eigenstates at two different $\xi$ points is defined in the most natural way as

$$
\begin{gathered}
e^{-i \Delta \varphi_{12}}=\frac{\left\langle\psi\left(\xi_{1}\right) \mid \psi\left(\xi_{2}\right)\right\rangle}{\left|\left\langle\psi\left(\xi_{1}\right) \mid \psi\left(\xi_{2}\right)\right\rangle\right|} \\
\Delta \varphi_{12}=-\operatorname{Im} \log \left\langle\psi\left(\xi_{1}\right) \mid \psi\left(\xi_{2}\right)\right\rangle
\end{gathered}
$$

This phase cannot have any physica meaning. In fact, any quantum mechanical state vector is arbitrary by a constant phase factor: "choice of the gauge" will be used in the following as a synonym for the (arbitrary) choice of such a phase. For instance, in numerical implementations the gauge is typically chosen by the diagonalisation routine. A simple change of gauge at $\xi_{1}$ and/or at $\xi_{2}$ will give the phase difference the most arbitrary value. Despite this, when one considers the total phase difference $\gamma$ along the closed path in the figure:

$$
\begin{aligned}
\gamma & =\Delta \varphi_{12}+\Delta \varphi_{23}+\Delta \varphi_{34}+\Delta \varphi_{41} \\
& =-\operatorname{Im} \log \left\langle\psi\left(\xi_{1}\right) \mid \psi\left(\xi_{2}\right)\right\rangle\left\langle\psi\left(\xi_{2}\right) \mid \psi\left(\xi_{3}\right)\right\rangle \\
\left\langle\psi\left(\xi_{3}\right) \mid \psi\left(\xi_{4}\right)\right\rangle\left\langle\psi\left(\xi_{4}\right) \mid \psi\left(\xi_{1}\right)\right\rangle &
\end{aligned}
$$

one immediately realizes that the phase difference is gauge invariant, since all the gauge-arbitrary phases cancel in pairs.

The most common Berry phase $\gamma$ is defined in the continuum limit. Suppose we have a smooth closed curve $C$ in the parameter domain, as in the figure, and we discretize it with a set of $N$ points, we can then assume that the gauge is chosen such that $|\psi(\zeta)\rangle$ is single-valued and varies in a differentiable way along the path. The phase difference between two contiguous points is therefore given by:
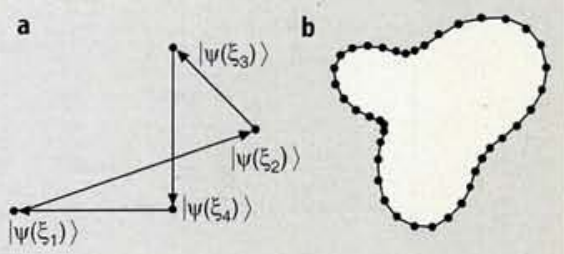

a. Four state vectors in parameter space, and a closed path connecting them. b. A smooth closed curve $C$ in parameter space, and its discretization.

$$
\begin{aligned}
e^{-i \Delta \varphi} & =\langle\psi(\xi) \mid \psi(\xi+\Delta \xi)\rangle \\
-i \Delta \varphi(\xi)|\psi(\xi+\Delta \xi)\rangle \mid & \approx\left\langle\psi(\xi) \mid \nabla_{\xi} \psi(\xi)\right\rangle \cdot \Delta \xi
\end{aligned}
$$

When the set of points becomes dense, the total phase difference $\gamma$ converges to the circuit integral of a real linear differential form, called the Berry connection:

$$
\gamma=\sum_{s=1}^{N} \Delta \varphi_{s, s+1} \rightarrow i \oint_{C}\left\langle\psi(\xi) \mid \nabla_{\xi} \psi(\xi)\right\rangle \cdot \delta \xi
$$

The above simple-minded algebra leads to a result of overwhelming physical importance, namely that a gaugeinvariant quantity is potentially a physical observable. Traditionally, we are accustomed to regarding any observable effect in quantum mechanics as the eigenvalue of some operator. The main message of Berry's milestone paper can be spelled out by saying that there are also observable effects of a completely different nature: the phase $\gamma$ cannot be expressed in terms of the eigenvalues of any operator, whereas it is by definition a gauge-invariant phase of the state vector. Several observables having the nature of a Berry phase have been found in different ares of physics: the macroscopic polarization of a crystalline dielectric is one of them, very recently discovered, but not the least by far.

Having understood that the Berry phase in general may provide a qualitatively novel quantum observable, one naturally wonders how and why this happens. To answer, one must re-examine the initial assumption of a parametric Hamiltonian, and realise its most fundamental meaning. In general, a quantum system having a parametric dependence in its Hamiltonian cannot be isolated: the parameter schematizes a kind of coupling with other variables not described by the given quantum Hamiltonian, or more generally with "the rest of the universe", to use Berry's words. The parametric Hamiltonian allows one to deal with a part of a larger system as if it were isolated: as a tradeoff, some observable effects may occur as gauge-invariant phases. 


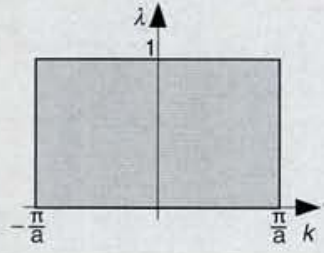

Fig. 5. The Berry phase $\gamma$ is evaluated along the contour of the rectangular domain in the $(k, \lambda)$ plane where $k$ is the Bloch vector in the one-dimensional Brillouin zone and $\lambda$ is a dimensionless scalar parameter which measures the amplitude of the ferroelectric distortion.

period $a$. A Schrödinger equation can be written in terms of $u$ : the quantum variable is $x$, whereas the Hamiltonian depends parametrically on $\mathrm{k}$ and $\lambda$. In order to apply the standard Berry phase formulation (see box) to this quantum system, it is sufficient to define a two-dimensional parameter $\xi$ whose two components are $k$ and $\lambda$. The curve $C$ is chosen as the contour of the rectangle in the $(k, \lambda)$ plane shown in Fig. 5, and the Berry phase $\gamma$ is evalu-ated in the usual way.

The polarization difference $\Delta P$ between the ferroelectric $(\lambda=1)$ and the centrosymmetric $(\lambda=0)$ structures coincides with the integrated current which flows through the linear chain (Fig. 4) while the ions are continuously displaced. According to the modern theory, this quantity is

$$
\Delta P=e \gamma / \pi+\Delta P_{\text {ion }}
$$

where $\mathrm{e}$ is is the electron charge, and $\Delta P_{i o n}$ is the trivial contribution of the classical ions (here polarization has the dimensions dipole per unit length).

The three-dimensional, multi-band analogue of Eq. (2) has been implemented in several first-principles calculations for real materials [1]. In the case of ferroelectric polarization - where a theory was not available - the numerical results display a quite spectacular agreement with the experimental data. The very first case study was $\mathrm{KNbO}_{3}$ in its tetragonal phase [3]: the theory predicts a spontaneous polarization $P_{\mathrm{S}}$ of $0.35 \mathrm{C} / \mathrm{m}^{2}$, compared with early measurements of about 0.30 $\mathrm{C} / \mathrm{m}^{2}$, and a recent one of $0.37 \mathrm{C} / \mathrm{m}^{2}$. Subsequent work on different materials has shown the same kind of agreement.

The theory for the macroscopic polarization of a crystalline dielectric based on the Berry phase of the electronic Bloch wavefunctions is much more than a mere computational tool. Focussing on the current instead of the charge is crucial to an unambiguous definition of what a physical observable really is. Standard textbook definitions for the macroscopic polarization - based on the periodic charge either are incorrect, or apply to extreme models only (Clausius-Mossotti). When periodic boundary conditions are adopted to describe the crystalline electrons in the usual way, the macroscopic polarization cannot be expressed as an operator expectation value: instead, it takes the form of a gauge-invariant phase of the occupied crystalline eigenstates. As such, it bears no relationship to the periodic charge density of the polarized crystal, where the density only depends on the square modulus of the occupied orbitals, so any phase information is lost.

\section{References}

[1] M.V. Berry, Proc. Roy. Soc. Lond. A 39245 (1984); Geometric Phases in Physics, Ed.: A. Shapere and F. Wilczek (World Scientific, Singapore 1989)

[2] R. Resta, Rev. Mod. Phys. 66809 (1994).

[3] R. Resta, Ferroelectrics 13651 (1992); R.D. KingSmith, D. Vanderbilt, Phys. Rev. B 471651 (1993); R. Resta, Europhys. Lett. 22133 (1993).

[4] R. Resta, M. Posternak, A. Baldereschi, Phys. Rev. Lett. 701010 (1993).

[5] R.M. Martin, Phys. Rev. B 51607 (1972).
The Swiss Federal Institute of Technology Lausanne (EPFL) invites applications for the dual position of

\section{PROFESSOR of PLASMA PHYSICS \\ and}

\section{DIRECTOR OF THE CENTRE DE RECHERCHES EN PHYSIQUE DES PLASMAS (CRPP)}

The CRPP is a research center of the EPFL employing about 120 people including $\mathrm{PhD}$ students. The main areas of activity are in plasma physics. The fusion related work is an integral part of the European Fusion Programme to which Switzerland participates. The main plasma physics installation at the EPFL is a 1 MA mid-size tokamak TCV (tokamak à configuration variable), which is being equipped with a 4.5 MW ECRH system. As full professor, the candidate is expected to participate in teaching courses and supervising diploma work and $\mathrm{PhD}$ thesis work. In addition to the managerial responsibilities of a director the candidate will lead the execution of the research programme of CRPP, including its participation in the international large programmes accessible through the European Programme and participate actively at least in one of the plasma physics research activity. Interest and ability to cooperate with other laboratories is required. Industrial spin-off activities from the research programme are strongly encouraged. The applicant must have a strong internationally recognized track record in research in the field of plasma physics, and demonstrated leadership of a research organisation. Experience in communicating and negotiating would be welcome. Starting date: as mutually convenient.

Applications from women are particularly welcome. For further information, please contact in writing:

Présidence de l'Ecole polytechnique fédérale de Lausanne, CE-Ecublens, CH 1015 Lausanne, Suisse
The Swiss Federal Institute of Technology Zurich (ETHZ) invites applications for a

\section{Professor for Quantum-Optics}

The Department of Physics covers teaching of basic and advanced courses at the undergraduate and graduate level at all divisions. The new professor is expected to teach diploma students in physics as well as to participate in the propaedeutical courses in physics at all divisions.

The duties of the new professor comprise mainly fundamental research in Quantum-Optics with applications in the visible, infrared or ultraviolet spectral range. $\mathrm{He} / \mathrm{she}$ is expected to build an attractive research group with a national and international reputation and to collaborate with research groups within the Institute of Quantum Electronics, neighbouring chairs and outside the ETHZ as well.

Applicants are expected to have experience in research in Quantum-Optics, present own important scientific contributions, and to be talented teachers.

Please submit your application together with a curriculum vitae and a list of publications to the President of ETH Zurich, Prof. Dr. J. Nüesch, ETH Zentrum, CH8092 Zurich, no later than April 30, 1997. The ETHZ specifically encourages female candidates to apply with a view towards increasing the proportion of female professors. 